\title{
Konnektivitätsveränderungen im O̊PNV- Netz durch die Einführung eines autonomen Shuttlebusses
}

\author{
Ludger Jürgens
}

Der Autor untersucht die Auswirkungen der Einführung eines autonomen Shuttlebusses auf die Angebotsseite. Die grundlegende Frage ist, ob sich das ÖPNV-Angebot aufgrund der Ergänzung eines vorhandenen ÖPNV-Netzes durch einen autonomen Shuttlebus signifikant steigern lässt und den Einwohnern Bad Birnbachs somit vielfältigere Möglichkeiten offenbart. Insbesondere geht er der Frage nach, ob und in welcher Art und Weise sich mit einem autonomen Shuttlebus die Erste-/Letzte-Meile-Problematik lösen lässt, die den konservativen ÖPNV bereits seit mehreren Jahren beschäftigt. Gerade diese Streckenabschnitte sind oftmals zu eng oder ungünstig gelegen, um mit einem konservativen Omnibus bedient zu werden. Daher stellen autonome Shuttlebusse eine sehr gute Ergänzung für diesen Bereich dar.

\subsection{Autonomer Shuttlebus als sinnvolle Ergänzung im ÖPNV- Netz?}

Aufgrund der dispersen Siedlungsstruktur mit schwach ausgeprägten Bevölkerungsschwerpunkten verteilt sich die Bevölkerung auf eine große Fläche. Die daraus resultierende geringe Bevölkerungsdichte führt dazu, dass innerhalb der Einzugsbereiche von Haltestellen im Öffentlichen Personennahverkehr (ÖPNV) eine geringere Anzahl an potenziellen Fahrgästen wohnt und somit das Nachfragepotenzial geringer ist, als dies im dichter besiedelten städtischen Umfeld der Fall ist.

Das Angebotsnetz der öffentlichen Verkehrsangebote in diesen Räumen wird häufig durch eine Kombination aus Schienenpersonennahverkehr (SPNV) und ÖPNV gebildet,

L. Jürgens $(\bowtie)$

DB Regio Bus Region Bayern, Ingolstadt, Deutschland

E-Mail: ludger.juergens@deutschebahn.com 
welche nicht zu jeder Tageszeit und nicht an jedem Ort gleichermaßen Verkehrsmöglichkeiten bieten. Die Disparitäten sind auf unterschiedliche ökonomische und logistische Faktoren zurückzuführen.

In der Regel gestaltet sich eine Bündelung der Verkehrsnachfrage im ÖPNV im ländlichen Raum schwierig, da durch eine räumliche und zeitliche Diversifizierung der Fahrtwünsche nicht alle Verkehrsströme auf einen zentralen Ort ausgerichtet sind. Häufig müssen verschiedene Orte für verschiedene Erledigungen aufgesucht werden und auf Grund unterschiedlicher Öffnungszeiten, Arbeitszeiten oder individueller Vorlieben besteht der Wunsch nach zeitlich flexibler Mobilität.

Vergleichsweise gut bündeln lassen sich die Schülerverkehre. Daher ist das ÖPNVAngebot im ländlichen Raum sehr stark auf den Schülerverkehr ausgerichtet, zumal Schüler auch die Hauptnutzergruppe darstellen (Steinrück und Küpper 2010, S. 17). Die auf die Bedürfnisse von Schülern ausgelegten Linienverkehre sind somit das Rückgrat des ÖPNVAngebots im ländlichen Raum. Sie repräsentieren jedoch auch ein Grundangebot für potenzielle weitere Fahrgäste - mit zeitlich und räumlich sehr starken Restriktionen. Ergänzend hierzu gibt es dort, wo ausreichend Nachfrage besteht, weitere ÖPNV-Angebote.

Ein weiteres Manko im öffentlichen Personennahverkehr ist die häufig fehlende Abstimmung der Verkehrsmittel an den Umsteigepunkten. Dies kann sowohl bei Umstiegen zwischen zwei Buslinien, als auch beim Umstieg zwischen SPNV und ÖPNV (und anders herum) vorkommen, wobei die fehlende Abstimmung verschiedene Ausprägungen annehmen kann. Beispielhaft wären dazu die nicht angepassten Abfahrtszeiten oder aber auch die fehlende Anbindung von Bahnhaltepunkten zu nennen. Durch dieses typische Erste-/ Letzte-Meile-Problem im ländlichen Raum, das meist durch einen außerhalb des Hauptortes liegenden Bahnhaltepunkt oder Bahnhof entsteht, ergeben sich oft Konnektivitätslücken im ÖPNV-Netz, d. h. ein Ort ist nicht ausreichend über den Tag hinweg mit den umliegenden Orten und Zentren verbunden.

Es wird angenommen, dass autonome Shuttlebusse eine wirksame Lösung darstellen, um diese Lücken schließen zu können. Diese Fahrzeuge können durch Einsparungen bei den Betriebs- und Personalkosten Strecken im ÖPNV bedienen, die bisher im (nichtautonomen) ÖPNV ökonomisch und ökologisch nicht sinnvoll leistbar sind.

\subsection{Fragestellung}

Inwieweit ein autonomer Shuttlebus dazu beitragen kann, die erwähnten Konnektivitätslücken zu schließen und welchen Effekt dieser Lückenschluss für das gesamte Angebotsnetz des ÖPNV hat, stellt die Hauptfragestellung dieser Untersuchung dar.

Die Verbindung zwischen dem Bahnhof von Bad Birnbach im Ortsteil Leithen und dem (Kur-)Zentrum von Bad Birnbach soll in dieser Untersuchung als Beispiel für einen Fall der Erste/Letzte Meile-Problematik dienen. In diesem konkreten Fall ist der Bahnhof rund zwei Kilometer vom Ortskern entfernt und mit dem bisherigen ÖPNV von der Ortsmitte aus nur sehr selten auf direktem Weg zu erreichen. Auf Teilen eben jener Strecke wird seit 
Oktober 2017 ein autonomer Shuttlebus auf einer ÖPNV-Linie betrieben und soll noch im Jahr 2019 die gesamte Strecke vom Neuen Marktplatz bis zum Bahnhof bedienen.

Um diese Bewertung vornehmen zu können, muss die Angebotsstruktur durch ein geeignetes Maß hinsichtlich der Konnektivität analysiert werden. Hierzu wird der Vergleich der Konnektivitätswerte vor und nach der Einführung eines autonomen Shuttlebusses als entscheidende Größe betrachtet. Insbesondere unter dem Gesichtspunkt der vorliegenden Erste-/Letzte-Meile-Problematik für den Fall Bad Birnbach sollen in dieser beispielhaften Berechnung Rückschlüsse gezogen werden können, ob die Einführung von autonomen Bussen zu diesem Zweck sinnvoll erscheint.

\subsection{Untersuchungsgebiet}

Für die funktionale Abgrenzung eines Untersuchungsgebiets für die Erreichbarkeit und Konnektivität eines Punktes innerhalb eines Verkehrsnetzes sind weder administrative Grenzen noch Verkehrsgemeinschaften eine sinnvolle Einteilung. Da der öffentliche Verkehr im ländlichen Raum vor allem eine Versorgungsfunktion erfüllt, ist das Heranziehen des Zentrale-Orte-Systems sinnvoll.

Der Markt Bad Birnbach liegt laut Regionalplan der Region Landshut (Regionaler Planungsverband Landshut 2007) im Mittelbereich des Mittelzentrums Pfarrkirchen (siehe Abb. 4.1). Das auf diese Weise definierte Untersuchungsgebiet umfasst insgesamt sieben Gemeinden, drei Märkte (Bad Birnbach, Tann und Triftern) sowie die Stadt Pfarrkirchen. In diesem Gebiet leben 43.464 Einwohner (Stand 30.06.2018) und es ist durch die SPNVAchse der Rottalbahn (Kursbuchnummer 946) mit vier Haltepunkten sowie rund 30 ÖPNV-Linien geprägt.

Das SPNV-/ÖPNV-Netz im Untersuchungsgebiet ist definiert durch die Strecke der Rottalbahn, die die Achse Bayerbach - Bad Birnbach - Anzenkirchen - Pfarrkirchen (und weiter nach Eggenfelden) erschließt. Hinzu kommen Busverbindungen, die größtenteils die Kreisstadt Pfarrkirchen oder den Markt Bad Birnbach als Ziel haben. Zu nennen sind hier vor allem die Verbindungen Pfarrkirchen - Johanniskirchen (Linie 6215), Pfarrkirchen - Arnstorf (Linie 6213), Pfarrkirchen - Egglham (Linie 6214) sowie die Linien 6205, 6217 sowie 7541 von Pfarrkirchen in Richtung Simbach am Inn. Die Umsteigemöglichkeit zwischen den Verkehrsmitteln Bahn und Bus ist hauptsächlich am Bahnhof Pfarrkirchen gegeben, da die weiteren Bahnhaltepunkte im Untersuchungsgebiet nur sehr rudimentär von Buslinien angefahren werden.

Aufgrund der nur lokalen Funktion und der geringen Effekte auf die Untersuchung wurden der Stadtbus Pfarrkirchen und die Stadtverkehre Pfarrkirchen für die Betrachtung nicht berücksichtigt. Auch der im April 2018 eingeführte „RuBi““, der als Ruf Bus in vier Sektoren im Gebiet um Bad Birnbach verkehrt (Bad Birnbach 2018), findet in der Untersuchung keine Berücksichtigung. 


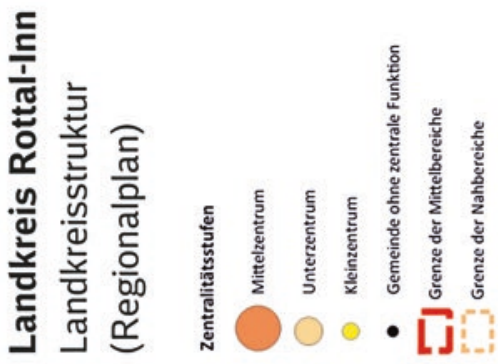
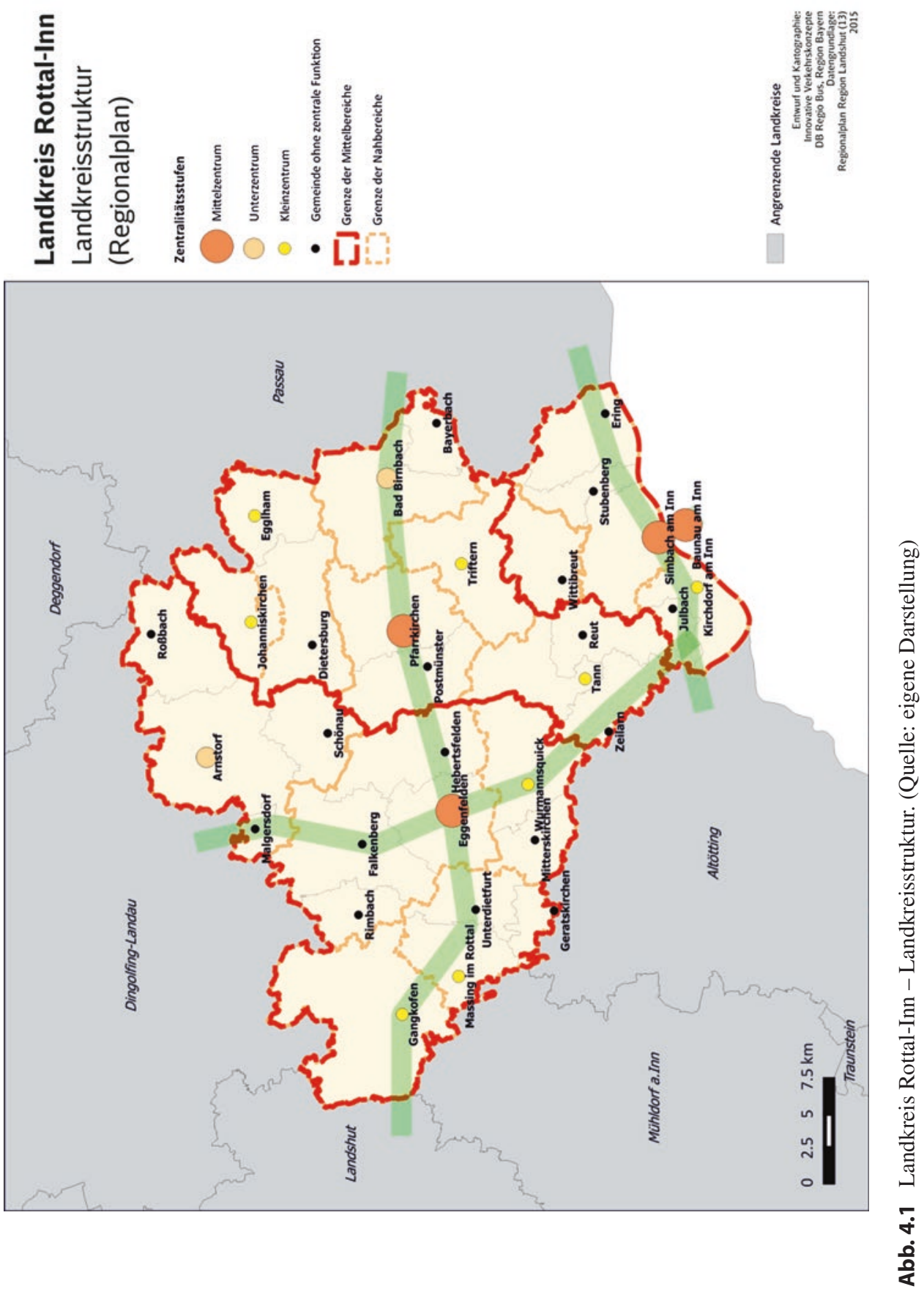


\subsection{Vorgehen bei der Erfassung und Bewertung der Konnektivität}

Es wurden alle SPNV- und ÖPNV-Linien des Untersuchungsgebiets hinsichtlich Fahrplänen, Haltestellen und genauer Linienführungen erfasst und mithilfe eines Geoinformationssystems (GIS) verarbeitet. Durch diesen Arbeitsschritt wurde im GIS ein Netz innerhalb des Untersuchungsraums aufgebaut, das alle möglichen Verbindungen (Kanten) zwischen den einzelnen Haltestellen (Knoten) darstellt. Zur Bewertung der Konnektivität wurde dieses Netz um die Informationen der Fahrtenhäufigkeit und der Linienanzahl zwischen zwei Haltestellen erweitert.

Die Linienanzahl (L) zwischen den Haltestellen gibt hierbei die Anzahl der zwischen den Haltestellen verkehrenden Buslinien an. Um die Fahrtenhäufigkeit eines Linienteilstücks zwischen zwei Haltestellen zu bewerten, wurden die Anzahl der Fahrten zwischen diesen Haltestellen in beiden Richtungen aus dem Fahrplan ausgelesen und zum Wert eines durchschnittlichen Tages (V) zusammengefügt. Der Wert eines durchschnittlichen Tages setzt sich wie in Formel 4.1 dargestellt zusammen.

$$
V=\frac{\sum \text { Fahrten } S+\sum \text { Fahrten } F+\sum \text { Fahrten } S a+\sum \text { Fahrten So }}{365}
$$

Mit

Fahrten $\mathrm{S}=$ Fahrten an Schultagen

Fahrten $\mathrm{F}=$ Fahrten an Ferientagen

Fahrten $\mathrm{Sa}=$ Fahrten an Samstagen

Fahrten So = Fahrten an Sonn- und Feiertagen

Unter Konnektivität wird allgemein ein Maß bezeichnet, welches die Einbindung von Knoten in ein Netzwerk wiedergibt (Möller und Kuschke 2015, S. 85). Wenn im Zusammenhang dieser Untersuchung von Konnektivität die Rede ist, wird diese als Maß für die Abhängigkeit eines Knotens von einem zuvor festgelegten Startknoten definiert. In diese Berechnung fließen die Variablen der Linienzahl (L) und der Anzahl der Fahrten zwischen zwei Haltestellen in beide Richtungen (V) als Ausstattungsindikatoren in die Konnektivitätsbewertung mit ein. Demnach entscheidet vorrangig die Lagegunst einer Haltestelle gegenüber der festgelegten Starthaltestelle über das Maß an Konnektivität (Schwarze 2005, S. 9).

Zur Untersuchung der Konnektivität des ÖPNV-Netzes im Untersuchungsgebiet wird eine Abwandlung des Bellmann-Ford-Algorithmus angewendet (Krumke und Noltemeier 2009, S. 182). Hierzu werden den Kanten jedoch nicht wie bei anderen Modellen zur Berechnung des kürzesten Pfades die Fahrdistanz (d) oder Fahrzeit (t) zugeordnet, sondern die oben aufgeführten Ausstattungsindikatoren ( $\mathrm{L}$ und V). Weiterhin wird für die Untersuchung angenommen, dass die Konnektivität der einzelnen Haltestellen zum Startort mit 
jedem durchlaufenen Knoten abnimmt. Als Bezugswert des jeweiligen Knotens wird dazu der Nachbar-(Vorgänger-)Knoten mit dem höchsten Konnektivitätswert herangezogen.

Diese Beziehung zum Vorgängerknoten wird mit Formel 4.2 ausgedrückt.

$$
K_{n}=K_{n-1}^{\max } *\left(1-\frac{1}{L+V}\right)
$$

Mit

$K_{n}=$ betrachteter Knoten

$K_{n-1}^{\max }=$ Vorgängerknoten mit dem höchsten Konnektivitätswert

$L=$ Linienzahl zwischen den Knoten

$V=$ Verbindungszahl zwischen den Knoten

Für die Berechnung der Konnektivität wird dem Startpunkt (zentrale Haltestelle in Bad Birnbach) der Wert 100 zugeordnet, da hier die stärkste Beziehung zum Zentrum von Bad Birnbach (Neuer Marktplatz) im ÖPNV-Netz vorliegt. Durch Anwendung der Formel wird nun den benachbarten Knoten (Haltestellen) ein Wert zugeordnet, der sich aus dem Startwert und den Gewichtungen der Kanten (Verbindungen) ergibt. Entsprechend der Formel 4.2 ist es nicht möglich, dass der Nachfolgeknoten einen höheren Konnektivitätswert aufweist, als der Vorgängerknoten, es sei denn, der Wert ergibt sich aus einer Verbindung zu einem benachbarten Knoten mit einem höheren Wert.

\section{Beispiel}

In der Abb. 4.2a ist der Ausgangszustand der Berechnung dargestellt. Die Kanten haben entsprechend der ausgewerteten Fahrpläne ihre Gewichtungen $(\mathrm{L}+\mathrm{V})$ bereits zugeordnet. Dem Startpunkt (A) wird der Wert 100 zugeordnet.

Die Abb. 4.2b zeigt die Situation nach dem Durchlaufen der ersten Schleife des Algorithmus. Auf den Startwert wurde für die zwei vom Startpunkt abgehenden Kanten die Formel angewendet und die daraus folgenden Werte den Punkten B und C überge-

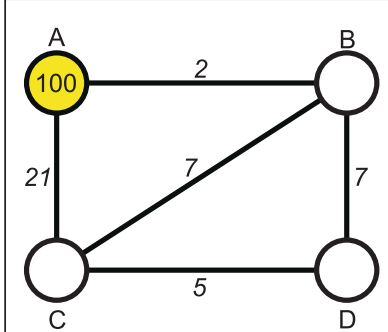

a) Ausgangszustand

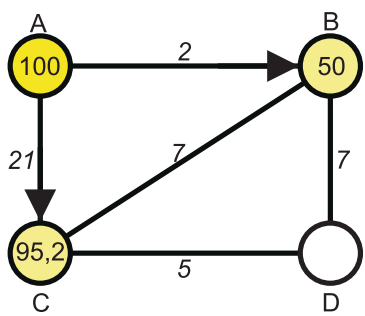

b) Situation nach dem ersten Durchlauf

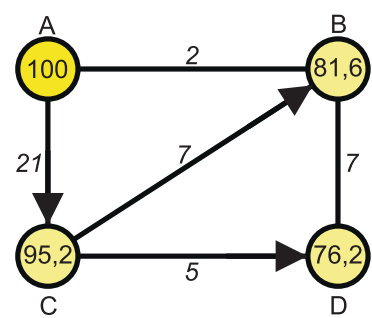

c) Situation nach dem zweiten Durchlauf

Abb. 4.2 Beispieldarstellung des angewendeten Algorithmus. (Quelle: eigene Darstellung) 
ben. Die Pfeile auf den Kanten zeigen dabei an, von welchem Punkt, bzw. über welche Kante, der jeweilige Punkt zugeordnet wurde.

Die Situation in Abb. 4.2c zeigt den Stand der Berechnung nach dem Durchlaufen der zweiten Schleife des Algorithmus. Dem Punkt D wurde hier bereits ein Wert zugeordnet, für den der Wert von Punkt C der Startwert ist und für den der Wert von der Kante zwischen Punkt C und D in der Formel angewendet wurde. Zudem wurde der Wert von Punkt B aus der zweiten Abbildung durch einen neuen Wert ersetzt, da dieser von Punkt $\mathrm{C}$ und den Kantenwerten der Kante zwischen Punkt B und C einen höheren Wert ergibt.

Die Formel für die Berechnung der Konnektivität zwischen den Knoten ist so gewählt, dass diese unabhängig vom Untersuchungsgebiet angewendet werden kann. Somit ist es möglich, die angewendete Methodik auch auf andere Untersuchungsfelder zu übertragen und diese miteinander zu vergleichen. Auch die Wahl der Starthaltestelle kann beliebig festgelegt werden. Somit ist mit dieser Methode nicht nur die Untersuchung der Konnektivität des ÖPNV-Netzes von Bad Birnbach ausgehend, sondern auch von anderen Orten nach Bad Birnbach durchführbar.

\subsection{Bewertung der Konnektivität der Angebotsstruktur ohne den autonomen Shuttlebus}

Im ersten Schritt soll die Angebotsstruktur des ÖPNV-Netzes zum Zeitpunkt vor der Einführung des autonomen Shuttlebusses betrachtet werden (Vorher-Situation). Hierzu werden das ÖPNV-Netz und die Verbindung der Rottalbahn im Untersuchungsgebiet erfasst und die entsprechend benötigten Daten (Linienzahl und Verbindungszahl) aus den Busund Bahnfahrplänen mit Stand Juli 2018 entnommen und in das GIS übertragen.

Für die Bewertung der Vorher-Situation im ÖPNV-Netz wird die Haltestelle Bad Birnbach, Feuerwehrhaus als Start- bzw. Bezugshaltestelle gewählt, da diese zwischen den beiden Zentren (Hofmark und Neuer Marktplatz) von Bad Birnbach liegt und fußläufig vom Neuen Marktplatz erreichbar ist. Als Starthaltestelle wird dieser der Wert 100 als Konnektivitätswert übermittelt. Mittels der oben aufgezeigten Berechnung ergibt sich das in Abb. 4.3 dargestellte Bild für die Haltestellen im Untersuchungsraum.

Allgemein ist festzustellen, dass die Konnektivitätswerte mit der Entfernung zur Starthaltestelle abnehmen. Vor allem abseits der Bahnstrecke der Rottalbahn ist ein rascher Abfall der Werte entlang der schwach getakteten Buslinien sichtbar. Wohingegen an der Bahnstrecke selbst und an den stärkeren Buslinien ab Pfarrkirchen eine deutlich geringere Abnahme der Konnektivitätswerte erkennbar ist. Neben den Haltestellen im Ortsgebiet von Bad Birnbach lässt sich insbesondere der Bahnhof in Pfarrkirchen als wichtiger Knotenpunkt für die Konnektivität nach Bad Birnbach benennen, der trotz der relativ weiten Entfernung mit 77,2 Punkte dennoch auf Rang 14 liegt. 


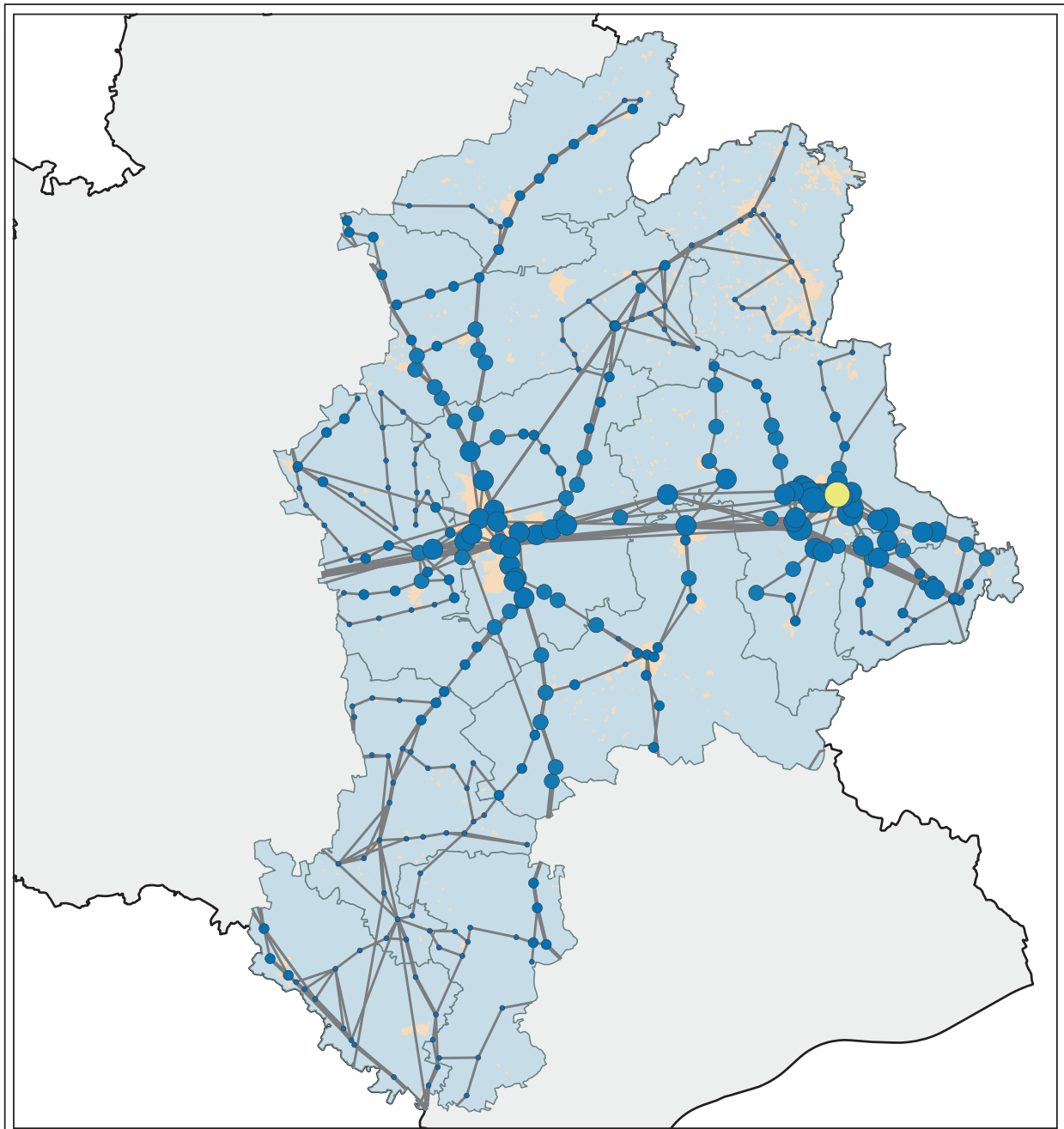

\section{Konnektivitätsveränderungen im ÖPNV-Netz} Netzkonnektivität - Vorher-Situation

Konnektivitätswerte

- $\quad 0$ - 19

- $20-39$

- $40-59$

$60-79$

$80-99$

Starthaltestelle
Verbindungswerte

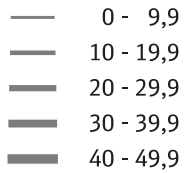

$40-49,9$
Untersuchungsgebiet

Siedlungsflächen im Untersuchungsgebiet

Landkreisgebiet
Entwurf und Kartographie: Ludger Jürgens, Inovative Verkehrskonzepte DB Regio Bus, Region Bayern Datengrundlage: Openstreetmap, eigene Berechnungen Januar 2019

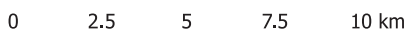

Abb. 4.3 Konnektivitätsveränderungen im ÖPNV-Netz (Vorher-Situation). (Quelle: eigene Darstellung) 
Auffällig ist vor allem, dass in Bayerbach (39,7 an der Haltestelle „Bayerbach, Ortsmitte“) und Egglham (11,8 an der Haltestelle „Egglham, Feuerwehrhaus“"), welche beide Nachbargemeinden von Bad Birnbach sind, eine vergleichsweise geringe Konnektivität feststellbar ist, obwohl hier eine geringe räumliche Entfernung vorliegt. Diese Werte lassen sich auf fehlende Direktverbindungen oder den fehlenden Anschluss der Bahnhöfe an das Busliniennetz begründen. Weiterhin sind auch im südlichen Untersuchungsgebiet geringe Werte in Bezug auf die Konnektivität nach Bad Birnbach vorhanden, die aufgrund der Umsteigenotwendigkeit in Pfarrkirchen ein Stück weit vorherzusehen waren.

Im Detailausschnitt für den Ortskern von Bad Birnbach in Abb. 4.4. liegen die Konnektivitätswerte zwischen 20,8 (Haltestelle „Bad Birnbach, Hochkreuz“) und 100 an der Starthaltestelle „Bad Birnbach, Feuerwehrhaus“. Wie bereits in Abb. 4.3 lassen sich die Abhängigkeiten zwischen der Stärke der Buslinien und den daraus resultierenden Konnektivitätswerten feststellen. Entlang der häufiger frequentierten Buslinien kann ein höherer Level der Konnektivitätswerte über eine größere Entfernung gehalten werden.

Ein gutes Beispiel, dass die räumliche Nähe in der Berechnung keinen Bezug auf die Berechnung der Konnektivität hat, ist die bereits genannte Haltestelle „Bad Birnbach, Hochkreuz“. Obwohl diese nur rund 300 Meter von der Starthaltestelle entfernt liegt, verfügt sie lediglich über einen Konnektivitätswert von 20,8. Dieser geringe Wert kommt dadurch zustande, dass zu dieser Haltestelle nur eine Linie mit durchschnittlich 0,35 Fahrten am Tag verkehrt.

\subsection{Bewertung der Konnektivität der Angebotsstruktur nach der Einführung des autonomen Shuttlebusses}

Nach der Vorher-Situation soll in einem zweiten Schritt die Nachher-Situation, d. h. nach der Einführung des autonomen Shuttlebusses und der entsprechenden Angebotserweiterung, berechnet werden. Hierzu gibt es im Vergleich zur Vorher-Situation kleine Änderungen im Berechnungsaufbau. So wird zum einen die Strecke des autonomen Shuttlebusses vom Neuen Marktplatz in Bad Birnbach zum Bahnhof Bad Birnbach mit aufgenommen, zum anderen wird neben der Haltestelle „Bad Birnbach, Feuerwehrhaus“ auch die Haltestelle „Bad Birnbach, Neuer Marktplatz“ als Start- bzw. Bezugshaltestelle für die Berechnung gesetzt. Im Einzelnen bedeuten diese Anpassungen, dass auch der Haltestelle „Bad Birnbach, Neuer Marktplatz“ der Startwert 100 übergeben wird und es somit zwei Starthaltestellen im Nachher-System gibt. Außerdem wird das Fahrtangebot des autonomen Shuttlebusses mit insgesamt 16 Fahrtenpaaren zum Bahnhof Bad Birnbach in die Berechnung mit aufgenommen. Nach der Umstellung der Startparameter wird die Berechnung, wie bei der Vorher-Situation, für das gesamte Untersuchungsgebiet vorgenommen.

In der Grundstruktur der Konnektivitätswerte in Abb. 4.5 fallen die Änderungen durch die Angebotserweiterung zunächst gering aus. Wenn man jedoch detaillierter auf die Werte der einzelnen Haltestellen eingeht, kann man feststellen, dass beim Großteil der Haltestellen der Konnektivitätswert gestiegen ist. In der Abb. 4.6 wird im Differenzbild der 


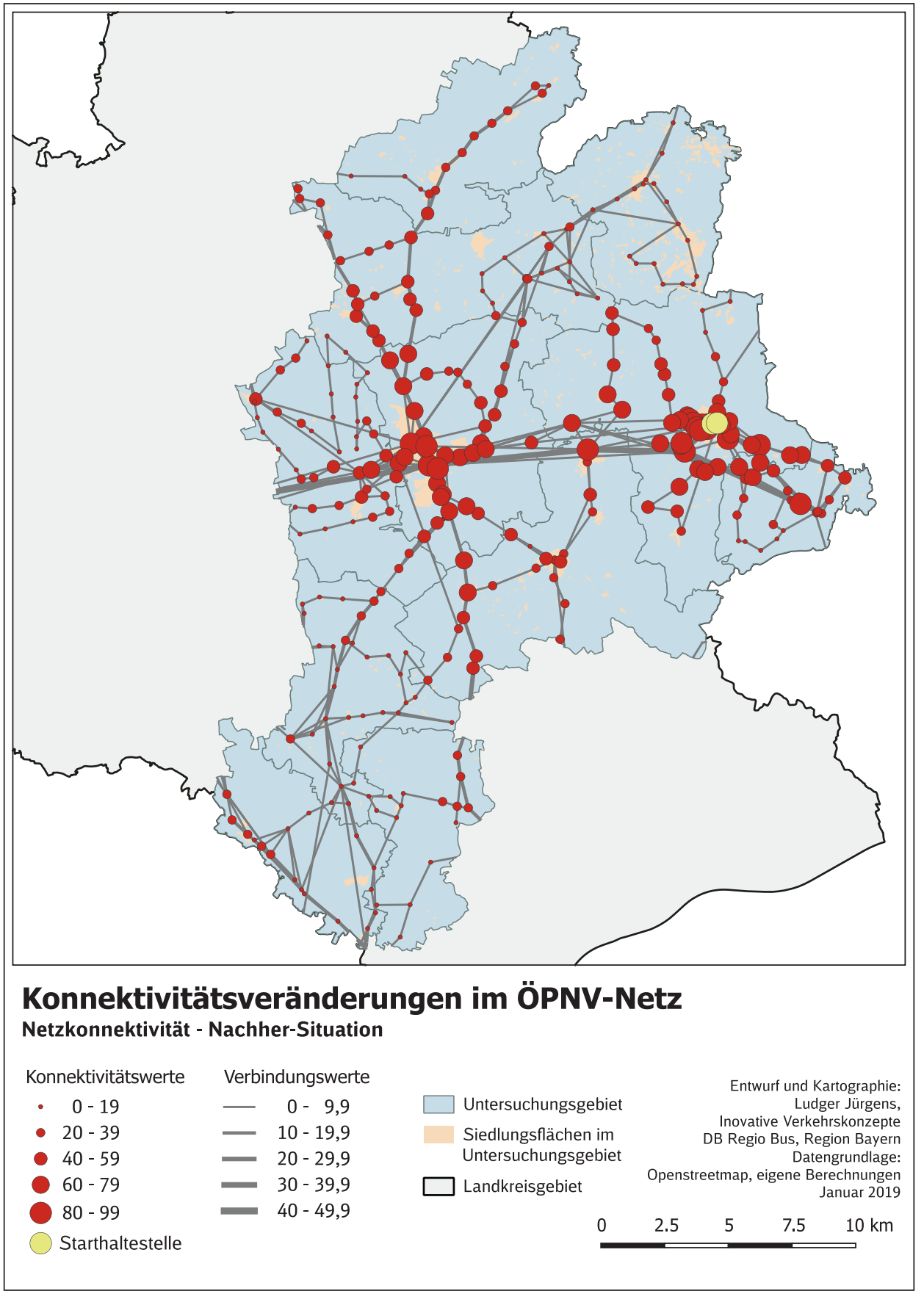

Abb. 4.4 Konnektivitätsveränderungen - Detailansicht (Vorher-Situation). (Quelle: eigene Darstellung) 


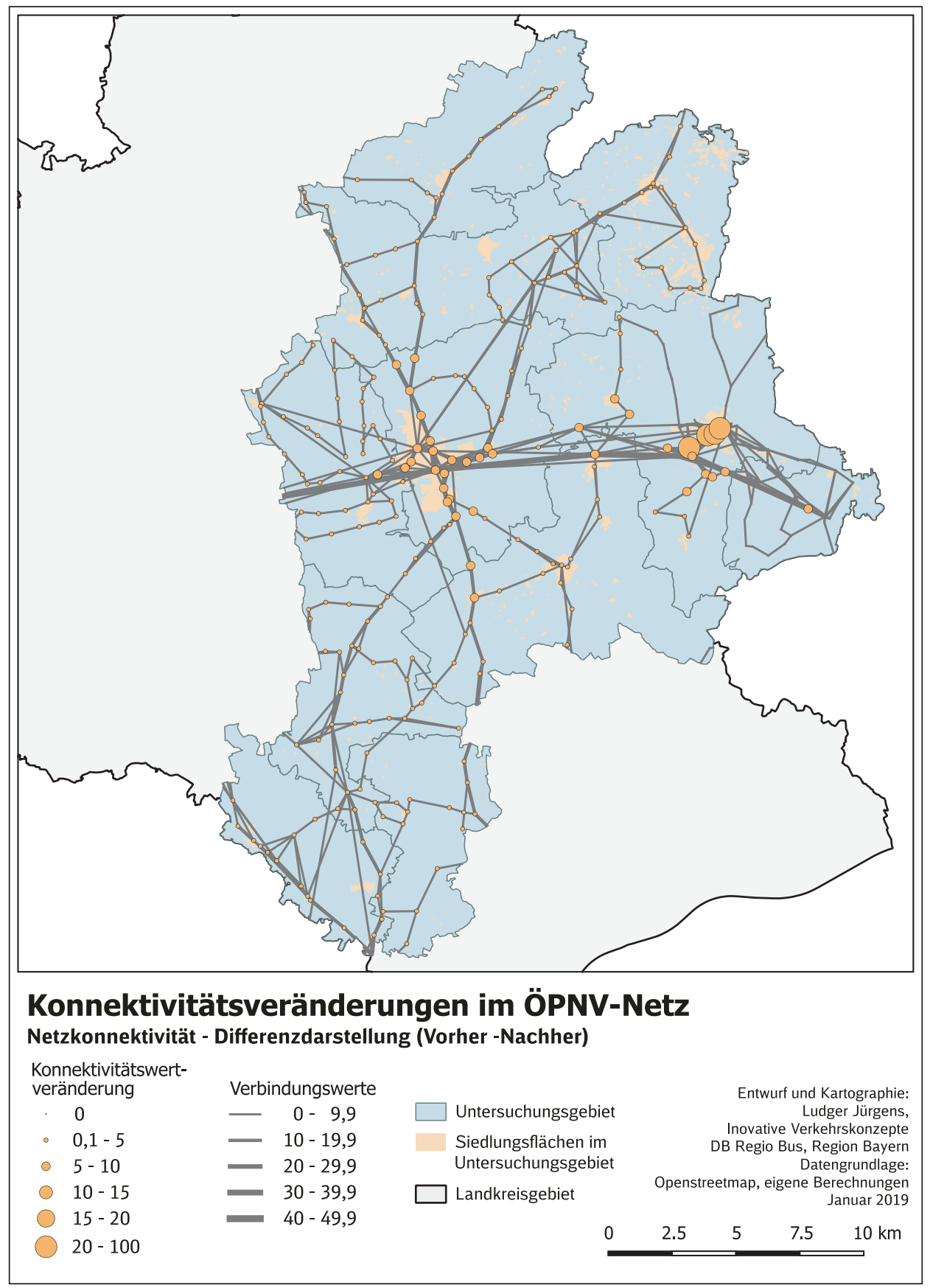

Abb. 4.5 Konnektivitätsveränderungen im ÖPNV-Netz (Nachher-Situation). (Quelle: eigene Darstellung) 


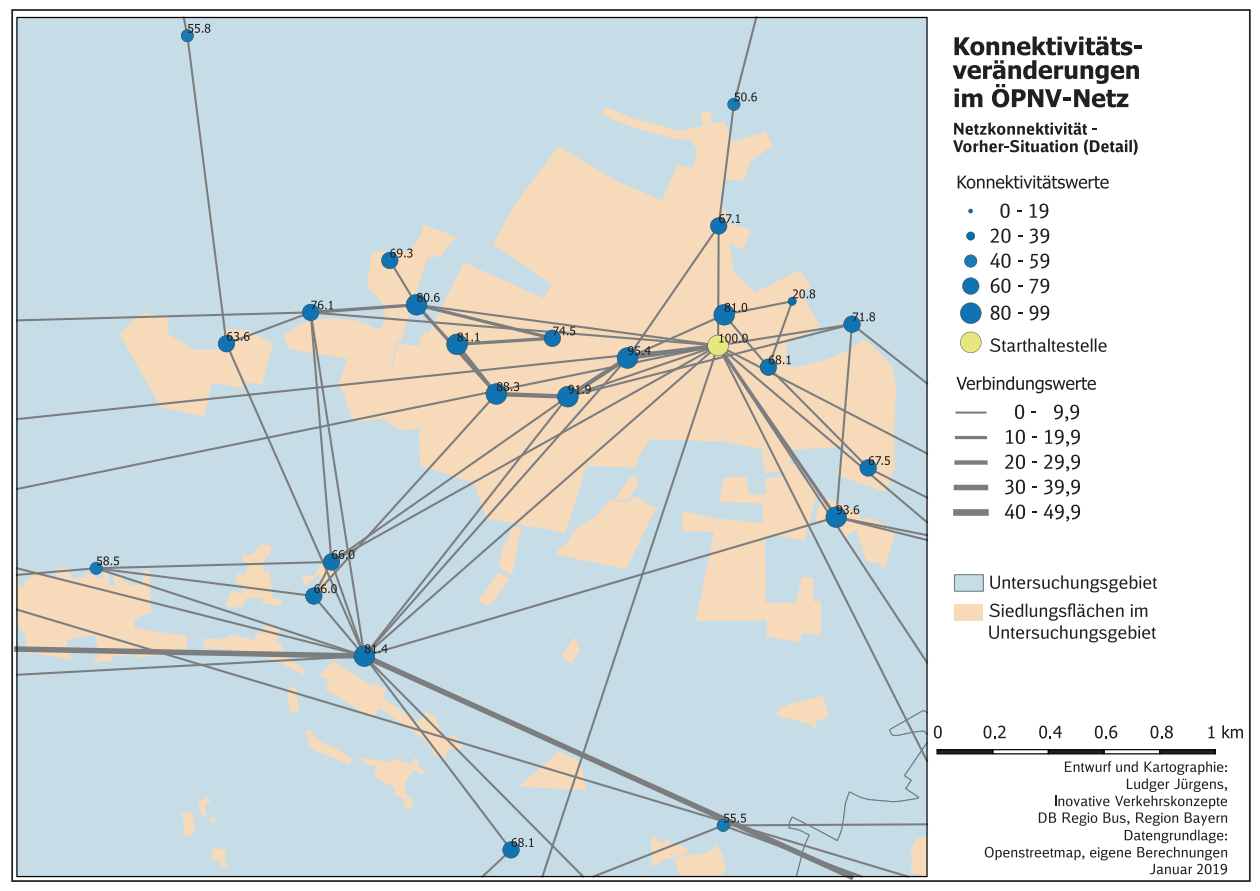

Abb. 4.6 Konnektivitätsveränderungen im ÖPNV-Netz (Differenzdarstellung). (Quelle: eigene Darstellung)

Vorher- und Nachher-Werte dargestellt, wie sich die Werte an den Punkten im Einzelnen verändert haben. Wie anzunehmen, sind dabei die Veränderungen ausschließlich positiv ausgeprägt, d. h. dass sich die Anbindung nach Bad Birnbach durch das Angebot des autonomen Shuttlebusses nachweislich verbessert hat.

In Abb. 4.6 lässt sich zudem erkennen, dass vor allem die Haltestelle „Gries“, die den Anknüpfungspunkt der Strecke des autonomen Shuttlebusses an das bestehende ÖPNVNetz darstellt, die höchste Konnektivitätssteigerung aufweist. Dieser Anstieg der Konnektivitätswerte zieht sich weiter zum Bahnhof Bad Birnbach und von dort aus entlang der Bahnstrecke und den davon abgehenden Buslinien in den großen Teil des Untersuchungsgebiets. Lediglich Teilräume im Norden und Südosten von Bad Birnbach weisen keinerlei Änderungen in den Ergebniswerten auf. In diesen Gebieten sind demnach keine Effekte durch die Angebotserweiterung durch den autonomen Bus festzustellen. Erklären kann man diese Tatsache damit, dass der Startpunkt „Bad Birnbach, Feuerwehrhaus“ direkt mit den Buslinien in diese Räume verbunden ist und somit der Steigerungseffekt ausbleibt. Im Raum südöstlich von Bad Birnbach, der größtenteils das Gemeindegebiet von Bayerbach darstellt, gibt es mit dem Bahnhof in Bayerbach nur eine einzelne Haltestelle, die eine Steigerung der Werte aufweist. Hier fehlt, wie am Bahnhof von Bad Birnbach, die Vernetzung zwischen dem SPNV und dem ÖPNV, da direkt am Bahnhof keine Bushaltestelle vorhanden ist, um entsprechende Effekte für die umliegenden Haltestellen zu erzeugen. 


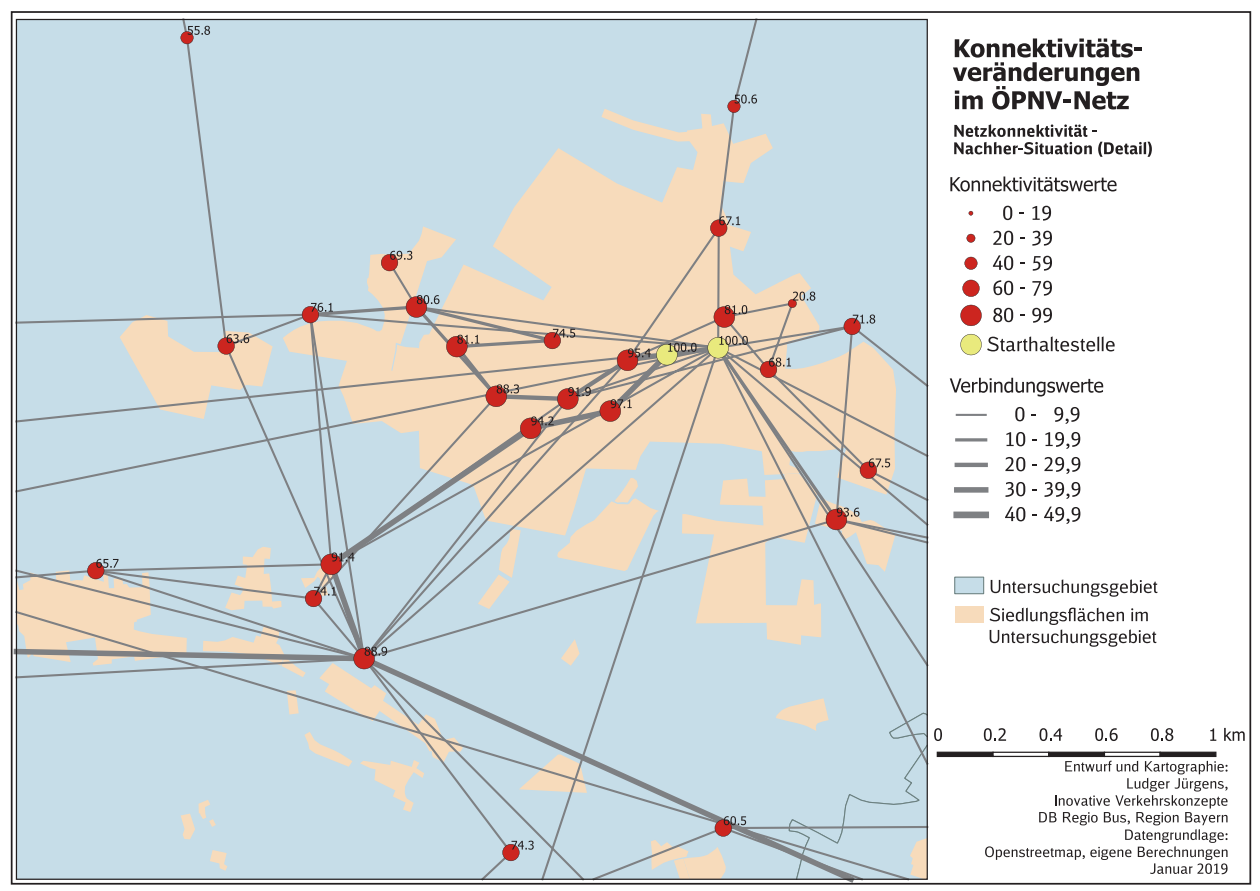

Abb. 4.7 Konnektivitätsveränderungen - Detailansicht (Nachher-Situation). (Quelle: eigene Darstellung)

In der Detaildarstellung in Abb. 4.7 haben sich am Intervall der Konnektivitätswerte aus den bereits genannten Effekten keine Änderungen ergeben. Dennoch lassen sich vor allem in Abb. 4.8 Veränderungen in den Werten südlich von Bad Birnbach feststellen. Sehr gut sind hier die neue Verbindung des autonomen Shuttlebusses und die deutliche $\mathrm{Zu}$ nahme der Konnektivität an der Haltestelle „Gries“ erkennbar. Anhand dieser Verbindung wird auch noch einmal sehr gut deutlich, dass die Effekte erst ab dieser Haltestelle Auswirkungen auf das bisher bestehende ÖPNV-Netz haben. Durch diese Tatsache und die vorhandenen guten Verbindungen innerhalb des Ortskerns von Bad Birnbach lassen sich auch die gleich bleibenden Werte im Ortskern erklären.

\subsection{Bewertung der Konnektivitätsveränderung auf die Angebotsstruktur durch die Einführung des autonomen Shuttlebusses}

Die Ergebnisse (Tab. 4.1) der Konnektivitätsveränderungen zeigen, dass die Auswirkungen der Einführung des autonomen Shuttlebusses, auf der Strecke zwischen dem Bahnhof Bad Birnbach und dem Ortszentrum, im gesamten Untersuchungsgebiet nachvollziehbar sind. In der Summe konnten die Konnektivitätspunkte in diesem Raum von vorher 9.991,41 


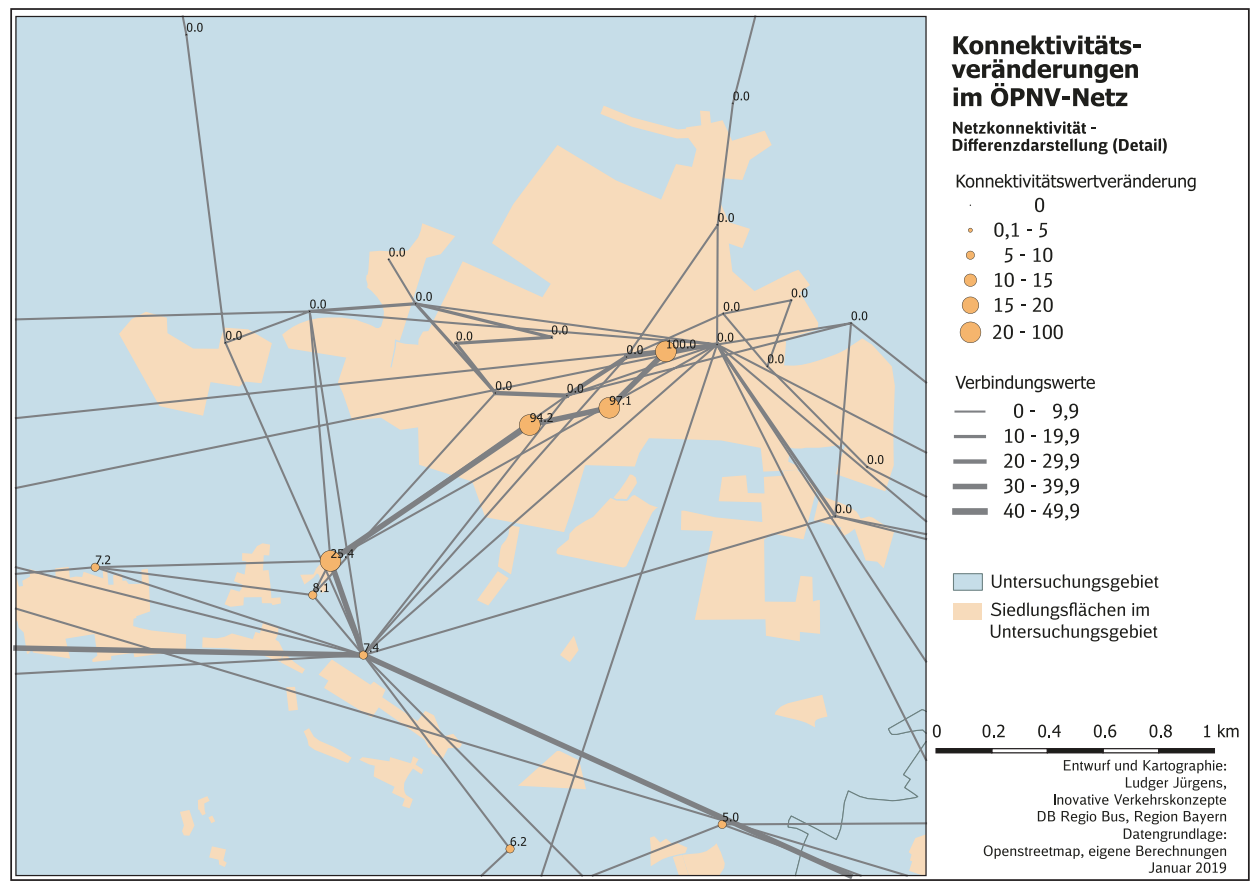

Abb. 4.8 Konnektivitätsveränderungen - Detailansicht (Differenzdarstellung). (Quelle: eigene Darstellung)

auf 10.963,11 gesteigert werden. Wenn man diesen Wert um die neu hinzugefügten Haltestellen reduziert, kommt man dennoch auf einen bereinigten Nachher-Wert von 10.671,84. Diese Steigerung von 971,63 (680,36 bereinigt) Konnektivitätspunkten im Untersuchungsraum entspricht einer prozentualen Steigerung von 9,72\% (bzw. 6,81\% in der bereinigten Berechnung). Demnach kann man die Aussage treffen, dass der Lückenschluss des Erste-/

Tab. 4.1 Haltestelle. Konnektivität vorher. Konnektivität nachher. Differenz

\begin{tabular}{l|l|l|l}
\hline Bad Birnbach Bahnhof & 81,44 & 88,86 & 7,42 \\
\hline Gries & 66,00 & 91,44 & 25,44 \\
\hline Bad Birnbach, VR-Bank & 95,38 & 95,38 & 0 \\
\hline Bayerbach, Bahnhof & 79,24 & 86,46 & 7,22 \\
\hline Bayerbach, Ortsmitte, & 39,72 & 39,72 & 0 \\
\hline Pfarrkirchen, Bahnhof/Busbahnhof & 77,19 & 84,22 & 7,03 \\
\hline Pfarrkirchen, Galgenberg & 68,38 & 74,61 & 6,23 \\
\hline Egglham, Rathaus & 7,92 & 8,64 & 0,72 \\
\hline Triftern, Marktplatz & 34,32 & 37,47 & 3,15 \\
\hline Tann, Busbahnhof & 16,58 & 18,09 & 1,51 \\
\hline Untersuchungsgebiet gesamt & 9991,48 & $10.963,11$ & 971,63 \\
& & $\left(10.671,84^{*}\right)$ & $\left(680,36^{*}\right)$ \\
\hline
\end{tabular}

*Werte um die neu eingeführten Haltestellen bereinigt 
Letzte-Meile-Problems zwischen dem Bahnhof Bad Birnbach und dem Ortszentrum von Bad Birnbach in Hinblick auf die Konnektivität und somit auch die Erreichbarkeit des Ortes Bad Birnbach für die anderen Orte im Untersuchungsgebiet nachweislich verbessert wurde. Wie bereits in den vorherigen Kapiteln beschrieben, nimmt hierbei insbesondere die gute Taktung der Rottalbahn eine wesentliche Rolle für die Verbreitung der gestiegenen Konnektivitätswerte ein. Den Anschluss an die Rottalbahn herzustellen und diese positiven Effekte hinzuzugewinnen, ist daher auch der größte Mehrwert durch die Einführung des autonomen Shuttles. Neben den bereits angesprochenen Haltestellen „Gries“ und „Bad Birnbach, Bahnhof“ ist der „Bahnhof/Busbahnhof“" in der Kreisstadt Pfarrkirchen ein weiterer wichtiger Knotenpunkt für die Ausstrahlung der Konnektivitätseffekte. An diesem Knoten kann eine Steigerung der Werte um 7,03 Punkte von 77,19 auf 84,22 festgestellt werden. Durch die hohe Verknüpfung dieses Knotens auf eine Vielzahl von Buslinien, wird dieser Differenzwert auch auf diese Linien bis in das Hinterland ausgestrahlt, was auch dort zu Steigerungen der Konnektivität in Bezug auf Bad Birnbach führt. Während westlich von Bad Birnbach die positiven Auswirkungen der neu geschaffenen Verbindung überwiegen, lassen sich im Ort Bad Birnbach und nördlich und südöstlich nur wenige Veränderungen in der Konnektivität feststellen. Auf diese Gebiete hat die Strecke des autonomen Busses, die sich zum Bahnhof orientiert, nur wenig oder gar keinen Einfluss. Hier sind die Buslinien, die den zweiten Startpunkt „Bad Birnbach, Feuerwehrhaus“ anfahren, auch weiterhin dominant, sodass die Übertragung der Steigerungseffekte hier ausbleibt. Mit der Haltestelle „Bayerbach, Bahnhof“ existiert zwar südöstlich von Bad Birnbach eine Haltestelle, die eine Steigerung um 7,22 Punkte von 79,24 auf 86,46 aufzuweisen hat, jedoch ist hier die fehlende Verknüpfung an das ÖPNV-Netz ausschlaggebend, dass sich dieser Steigerungseffekt nicht weiter ausbreitet.

\subsection{Fazit und Ausblick}

Die Untersuchung zeigt, dass autonome Shuttlebusse in der Lage sind, eine Entlastung für das Erste-/Letzte-Meile-Problem in ländlichen Räumen zu bieten. Bereits mit dem derzeitigen technologischen Stand können die Shuttlebusse auf passenden Strecken eine Lösung für eben diese Problematik sein.

Die positiven Veränderungen der Konnektivitätswerte zeigen zumindest für den Fall in Bad Birnbach auf, dass die Angebotserweiterung des autonomen Shuttlebusses über weite Strecken hinweg die Erreichbarkeit des Ortes Bad Birnbach erhöht und somit große Flächen des Untersuchungsgebiets besser anbindet. Die Untersuchung in Bad Birnbach bezieht sich, wie bereits erläutert, auf einen typischen Fall der Erste-/Letzte-MeileProblematik, dessen Verbindung über den ÖPNV bisher nicht hergestellt wurde. Da in der Studie noch einmal deutlich wurde, dass das Zusammenspiel zwischen dem autonomen Shuttlebus und der eng getakteten Rottalbahn eine entscheidende Rolle einnimmt, erscheint es umso wichtiger, dass dieser Lückenschluss endlich vollzogen wurde. 
Im Umkehrschluss bedeutet dies aber auch, dass durch die Einführung von autonomen Shuttlebussen nicht alle Mobilitätsherausforderungen im ländlichen Raum gelöst werden können. Allerdings können sie bei gezieltem Einsatz und guter Planung bereits jetzt einen Beitrag zur Verminderung der Erste-/Letzte-Meile-Problematik leisten. Durch zukünftige Verbesserungen der Technologie der autonomen Shuttlebusse können sich noch weitere Einsatzmöglichkeiten ergeben, durch die weitere Effekte der Verbesserung der Konnektivität möglich erscheinen.

Inwieweit die in der Studie ermittelten Effekte für Bad Birnbach auch auf andere Raumausschnitte übertragbar sind, kann zum gegenwärtigen Zeitpunkt nicht final erörtert werden. Jedoch können bei vergleichbaren Einsatzszenarien ebenfalls positive Konnektivitätseffekte erwartet werden. In welchem Umfang diese im Einzelnen ausfallen, müsste durch an diese Studie anknüpfende Betrachtungen ermittelt werden.

\section{Literatur}

Bad Birnbach (2018) Der Rubi fährt. https://www.badbirnbach.de/presse/der-rubi-faehrt. Zugegriffen: 12. Dezember 2019

Krumke SO, Noltemeier H (2009) Graphentheoretische Konzepte und Algorithmen. Springer-Verlag

Möller M, Kuschke V (2015) Konnektivität im Schienennetz der Deutschen Bahn. zfv - Zeitschrift für Geodäsie, Geoinformation und Landmanagement 2/2015: 85-90. doi https://doi. org/10.12902/zfv-0055-2015

Regionaler Planungsverband Landshut (2007) Regionalplan Region Landshut (13). Nah- und Mittelbereiche. http://www.region.landshut.org/plan/plan_aktuell/teil_a/a_karte_nahmittelbereiche. pdf. Zugegriffen: 8. Januar 2019

Schwarze B (2005) Erreichbarkeitsindikatoren in der Nahverkehrsplanung. (= Arbeitspapier 184). Dortmund: Institut für Raumplanung, Universität Dortmund.

Steinrück B, Küpper P (2010) Mobilität in ländlichen Räumen unter besonderer Berücksichtigung bedarfsgesteuerter Bedienformen des ÖPNV. Arbeitsberichte aus der vTI-Agrarökonomie $02 / 2010$

Open Access Dieses Kapitel wird unter der Creative Commons Namensnennung 4.0 International Lizenz (http://creativecommons.org/licenses/by/4.0/deed.de) veröffentlicht, welche die Nutzung, Vervielfältigung, Bearbeitung, Verbreitung und Wiedergabe in jeglichem Medium und Format erlaubt, sofern Sie den/die ursprünglichen Autor(en) und die Quelle ordnungsgemäß nennen, einen Link zur Creative Commons Lizenz beifügen und angeben, ob Änderungen vorgenommen wurden.

Die in diesem Kapitel enthaltenen Bilder und sonstiges Drittmaterial unterliegen ebenfalls der genannten Creative Commons Lizenz, sofern sich aus der Abbildungslegende nichts anderes ergibt. Sofern das betreffende Material nicht unter der genannten Creative Commons Lizenz steht und die betreffende Handlung nicht nach gesetzlichen Vorschriften erlaubt ist, ist für die oben aufgeführten Weiterverwendungen des Materials die Einwilligung des jeweiligen Rechteinhabers einzuholen.

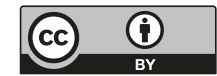

